\title{
Modélisation pluie-débit : Validation par simulation de la méthode DPFT
}

\author{
I. Nalbantis, Ch. Obled et Y. Rodriguez \\ Institut de mécanique, INPG, Grenoble
}

\section{Introduction}

La méthode de la DPFT (différence première de la fonction de transfert), développée à la Division technique générale de l'EDF et présentée par D. DuBAND (1978), est une extension de la méthode classique de l'hydrogramme unitaire. Elle suppose donc que, globalement sur un bassin, la pluie se transforme en débit par passage à travers 2 fonctions successives :

- une fonction de "production" qui transforme la pluie " brute " tombée sur le bassin en pluie " efficace " ruisselée. Cette fonction est généralement fortement non linéaire et dépend des conditions antérieures;

- une fonction de "transfert ", linéaire cette fois, qui étale dans le temps les débits issus d'une pluie efficace donnée.

L'hydrogramme unitaire (U.H.) s'est déjà vu proposer de nombreuses améliorations, essentiellement numérique, que l'on trouvera en partie chez J.C. Dooge (1973).

La méthode DPFT reprend certaines de ces améliorations mais surtout en propose d'autres. Sa version initial (DuBAND 1978, DuBand et GuIllot 1980) est décrite en détail dans le Guide de prévision des crues (P.A. RocHE et al. 1987). Elle a été appliquée avec succès à plusieurs dizaines de petits bassins (qq 100 à qq 1000 $\mathrm{km}^{2}$ ), mais des problèmes sérieux ont été rencontrés sur quelques-uns. Cela a occasionné quelques doutes sur sa généralité, sur sa convergence, et a limité, en conséquence, sa diffusion.

En collaboration avec la DTG, le groupe d'Hydrologie de l'IMG s'est donc proposé de lever ces doutes en analysant, et si possible en validant, la méthode. Malheureusement, il était exclu, sur des bassins réels, de connaître les "vraies " valeurs des pluies " efficaces " et de la "fonction de transfert ". Il n'est même pas sûr que ces notions correspondent strictement à la réalité. Notre objectif ne sera donc pas de vérifier la pertinence d'un modèle par rapport à la Nature mais de vérifier que ce modèle est identifiable dans les hypothèses qu'il s'est défini et qui sont couramment admises par ailleurs. C'est pourquoi nous déciderons de travailler en simulation.

Dans une première partie, nous donnerons quelques compléments sur la version de la DPFT utilisée, comportant les améliorations proposées récemment. Dans une seconde partie, nous la testerons sur des données simulées et un bassin fictif.

\section{La méthode DPFT}

\subsection{Mise en œuvre. Principe de l'algorithme}

La méthode se rapproche d'une méthode de gradient (VERSIANI, 1983) où, à partir d'une estimation initiale, on déterminera 2 jeux d'inconnues:

- les valeurs de la fonction de transfert $H(t)$ ou plutôt de sa différence première, entre pluie et variation de débit, soit $h(t)=H(t)-H(t-1)$. De manière discrète, on retiendra les $k$ valeurs $\left\{h_{1}, h_{2} \ldots h_{K}\right\}, K$ étant la " mémoire " du système;

- les pluies efficaces $P E_{i}$ correspondant à chaque pluie brute $P B_{i}$ non nulle.

Rain-flowrate modelization. A contribution to the development of the DPFT method

A whole set of algorithms seeking to improve the implementation and accuracy of the DPFT method has been proposed. In treatments concerning real data, the absence of exact reference values makes it difficult to discriminate between methods. It has therefore been decided to use data generated via a known model, whose inputs and outputs, as well as the intermediate data such as accurate rainfall and the transfer function, are known. Using accurate input and output data, or data noise-effected in a realistic manner, the most adapted algorithms, as well as the sensitivity of the method to various types of context or errors, have been able to be assessed. 


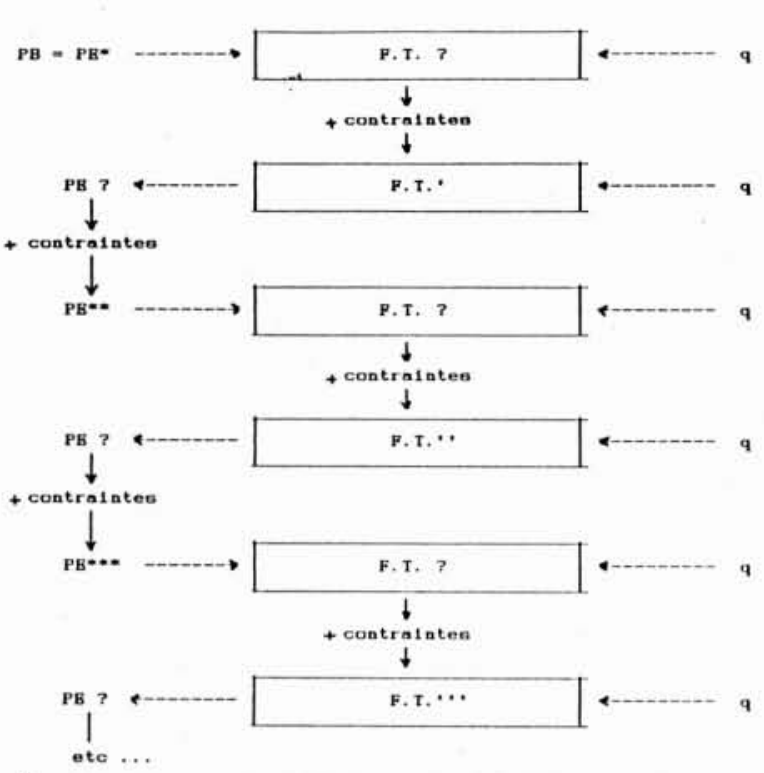

1. Algorithme itératif de la méthode DPFT. A chaque étape, le point d'interrogation indique la famille d'inconnues à calculer.

La méthode est itérative (figure 1). Pour l'initialiser, on suppose que les pluies brutes $P B$ constituent une première estimation acceptable des $P E$, et on calcule d'abord une première approximation de la fonction de transfert $H(t)$. On lui applique ensuite un certain nombre de traitements (contrainte de positivité, lissage éventuel, normalisation à 1$)$.

Puis, supposant $h(t)$ connue, on passe au calcul des pluies efficaces. Il s'agit là d'un problème de déconvolution assez délicat (retrouver l'entrée $P E$ du système à partir de la sortie $Q$ et d'un modèle $h(t)$ ).

Alors que la première étape considère d'emblée toutes les crues, (figure 2), la seconde s'effectue nécessairement crue par crue (figure 3). Là encore, on applique des contraintes qui font que les $P E$ ne peuvent être négatives, ni trop supérieures aux pluies brutes.

En fait, la méthode n'est pas une méthode de gradient dans la mesure où celles-ci ne prennent pas en compte les contraintes d'inégalité dans l'algorithme.

\subsection{Fonctionnement de la méthode}

On rappelle d'abord que, dans la méthode initialement proposée par D. DUBAND (1980), on commence par choisir la longueur $K$ de la fonction de transfert $H(t)$.

On résout ensuite globalement, en différences premières et sur l'ensemble des crues, le système d'équations :

$$
q(i, L)=\sum_{j=1}^{k} h_{j} \cdot P(i-j+1, L)+\varepsilon
$$

où $L$ indexe le $\mathrm{n}^{\circ}$ de la crue. On dispose alors de $N_{\text {crues }}$

$\sum N_{L}$ équations (une par débit disponible) à $K$ incon$L=1$ nues, $N_{L}$ étant le nombre de débits par crue.

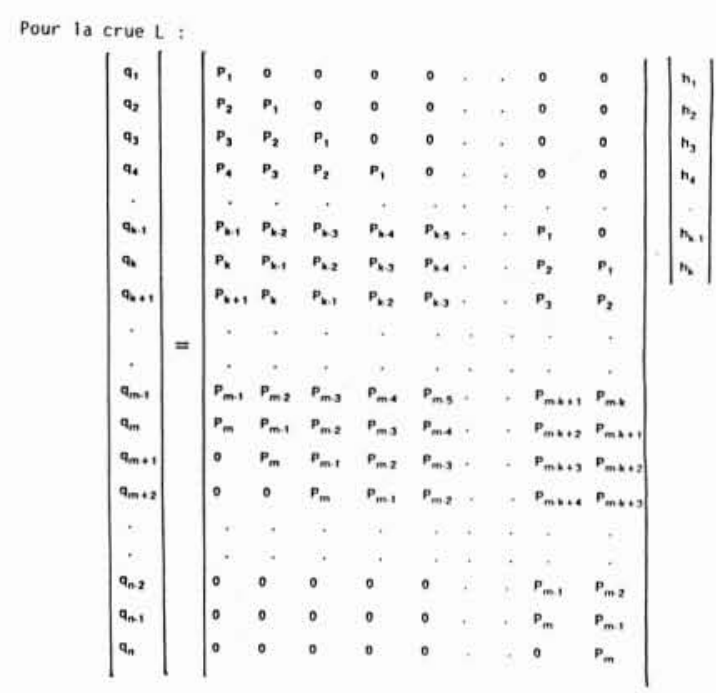

soit en notation matricielle :

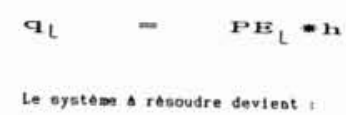

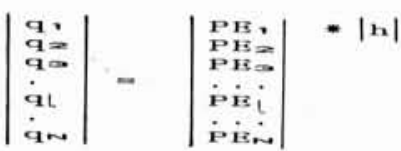

2. Système d'identification de la DPFT.

3. Système de déconvolution pour le calcul des pluies efficaces. Les $h_{j}^{*}$ prolongent, au delà du $k+1$ pas de temps, les $h_{i}$ identifiés. 
Remarquons que, pour chaque crue, il ne peut être pris en compte au plus que $K$ variations de débits suivant la dernière pluie efficace. En effet, pour la mémoire $K$ supposée, il n'y a plus d'effet de la pluie sur le débit au-delà de $K$ pas de temps.

Cela conduit à un problème "simple " de moindres carrés qui nécessite la solution d'un système de dimensions $K \times K$. Compte-tenu des oscillations parfois intempestives des $h_{k}$ ainsi calculés, alors que la fonction de transfert est supposée relativement lisse, surtout dans la phase de récession, on procède à un lissage, manuel ou automatique, des derniers coefficients.

En particulier, on reconstitue d'abord les $H_{i}$ à partir des $h_{i}$ qui sont au préalable mis à 0 s'ils sont numériquement négatifs, et dans l'optique de l'étape suivante : la déconvolution, on peut, voire on doit, introduire une notion de conservation des volumes (i.e. volume ruisselé, ou " efficace ", = volume écoulé). Ce qui nécessite:

$$
\sum_{i=1}^{K} H_{i} \# 1 \quad \text { ou } \quad \sum_{i=1}^{K} \sum_{j=1}^{i} h_{j} \# 1
$$

On passe ensuite à la $2^{\mathrm{e}}$ phase, plus délicate, de déconvolution des pluies. Celle-ci peut se faire de 2 façons : soit par calcul d'un terme correctif sur l'estimation précédente des pluies efficaces, soit par déconvolution directe. Bien que la première approche ait été utilisée initialement par D. DUBAND (1978), c'est la seconde que nous présenterons. Le système à résoudre peut encore s'écrire globalement mais en fait il dégénère bloc par bloc, chaque bloc correspondant à une crue (figure 3).

On constate ici que le système d'équations de la crue $L$, qui comporte $m_{L}$ pluies à identifier, ne peut comporter au maximum que $N_{L}=m_{L}+K-1$ équations.

Cela signifie que nous aurons $m_{L}$ "variables": $P E_{1} \ldots P E_{m L}$ pour au plus $m_{L}+K-1$ « observations " ou équations. Or, on sait que la stabilité des résultats est liée au nombre de degrés de liberté (i.e. nombre d'observations indépendantes moins nombre de paramètres à identifier). On peut donc s'attendre à des problèmes numériques parfois sérieux, et courants dans les techniques de déconvolution.

Une contrainte externe appliquée aux résultats est évidemment de vérifier que :

$$
P E \geqslant 0
$$

Mais on peut aussi imaginer d'aller plus loin. Par exemple, on peut exiger que globalement $\Sigma P_{E}(L) \#$ volume écoulé et qu'à chaque pas de temps $P_{E}<P_{B}$ (pluie brute) si celle-ci est connue avec précision.

Historiquement (cf. GuILLOT et DUBAND 1980), et pour des raisons pratiques, la méthode avait été programmée pour des épisodes de longueur fixe (en débit) avec des techniques de corrélation multiple, par correction successive des $P_{E}$ plutôt que par déconvolution directe, et avec une scrutation manuelle, à chaque pas, pour introduire ou non des contraintes.

\subsection{Variantes et améliorations proposées}

La première, qui s'impose dès que l'on peut surmonter les problèmes informatiques correspondants, est de ne plus travailler en épisode standard de longueur fixe, mais en épisodes variables, de longueur égale au nombre de pluies présumées efficaces $m_{L}$ augmentée de la mémoire $K \mathrm{du}$ bassin. Ceci nécessite quand même une gestion dynamique de tableaux et/ou de fichiers assez délicate.

A part cela, les problèmes, et donc les améliorations souhaitées, ont porté soit sur l'estimation de la FT, soit sur celle des $P E$. Nous allons les considérer successivement.

\subsubsection{Améliorations sur la F.T.}

a) On a d'abord reprogrammé le calcul de la F.T. en moindres carrés bruts (sans terme constant) plutôt qu'en corrélation multiple.

b) On a ensuite pris en compte, dans l'algorithme, la contrainte de conservation des volumes ruisselé/écoulé. Cette idée, décrite dans Versiani (1983) a été progrémme et consiste à identifier les $H_{j}$ de la F.T. sous la contrainte $\Sigma H_{j}=1$.

On espérait ainsi avoir une solution plus optimale qu'en imposant la contrainte dans un deuxième temps. Par contre, les contraintes d'inégalités $H_{j}>0$ n'ont pu être introduites simplement à ce niveau.

c) Identification de la F.T. par modélismation ARMAX.

Les coefficients $h_{j}$ ou $H_{j}$ obtenus terme à terme donnent souvent une forme en ligne brisée à la F.T., alors que certaines considérations conceptuelles fournissent ou justifient des formes lisses. Au lieu d'effectuer un lissage a postériori, on a remplacé l'étape de calcul des $H_{j}$ par le calage d'un modèle ARMAX (BASTIN, 1984, DELLEUR et OBLED, 1985), de la forme :

$$
q_{i}+\sum_{j=1}^{m} a_{j} \cdot q_{i-j}=\sum_{k=0}^{n} b_{k} \cdot P E_{i-k}+e_{i}
$$

qui présente divers avantages:

- le nombre de coefficients à identifier $(m+n)$ peut être significativement inférieur à $K$, celui de l'identification terme à terme, d'où un avantage de parcimonie; - en fait ici, la mémoire est théoriquement infine, ce qui peut permettre ensuite de mieux choisir $K$ quand les $H_{i}$ s'annullent;

- et surtout, on peut obtenir les $H_{i}$ de la F.T. par une division de polynômes : si on note $z^{-1}$ l'opérateur retard, tel que $z^{-1} \cdot P_{i}=P_{i-1}$ et $z^{-k} \cdot P_{i}=P_{i-k}$, alors le modèle ARMAX s'écrit :

$$
\begin{aligned}
H\left(z^{-1}\right)=h_{1}+h_{2} z^{-1}+\ldots & h_{K} z^{-K}+\ldots \\
& =\frac{b_{0}+b_{1} z^{-1}+\ldots b_{i} z^{-n}}{1+a_{1} z^{-1}+\ldots a_{m} z^{-m}}
\end{aligned}
$$

et fournit automatiquement un résultat très lisse (pour $m$ et $n$ petits).

Un inconvénient est la nécessité de fixer $a$ priori $m$ et $n$, ou de les optimiser par tâtonnement.

\subsubsection{Sur l'estimation des pluies efficaces}

a) Comme pour la F.T., on a programmé l'algorithme en moindres carrés stricts plutôt qu'en corrélation multiple. 
D'autre part, le calcul des pluies efficaces peut se faire d'une part en déconvolution directe (cf. 1.3), d'autre part en corrections successives (GuILloT et DUBAND, 1980).

b) Néanmoins, on a continué à constater, dans les pluies efficaces estimées, la présence d'oscillations manifestement dues à des instabilités. Si l'on se rappelle que ces pluies efficaces sont calculées pratiquement comme des coefficients de régression dans une corrélation multiple, on peut penser aux techniques existantes pour réduire les fluctuations de ces coefficients. Celles-ci sont souvent dues à l'intercorrélation des variables explicatives correspondantes. Une technique, assez lourde à mettre en œuvre, consiste à les orthogonaliser (Guillot et Duband, 1980).

c) Une autre, connue sous le nom de "régression en crête " ou Ridge Regression (HoERL et KENNARD, 1980) consiste à perturber légèrement le système linéaire à résoudre. Cela éloigne légèrement de l'optimum recherché mais en améliorant considérablement le conditionnement. C'est la technique qui a été retenue. Déjà testée par B. VERSIANI (1983) sur données réelles, elle avait réduit significativement les oscillations rencontrées avec la méthode de base.

d) Ces méthodes restent cependant du type moindres carrés, avec les limitations que cela comporte pour la prise en compte des contraintes, surtout sous forme d'inégalités. D'où l'idée de tenter la programmation linéaire pour calculer les pluies efficaces.

Sans rappeler en détail cette technique, disons qu'elle permet d'entrer dans l'algorithme les contraintes :

$P E(i, L)>0$ quelque soit $i$ et la crue $L$. Le critère consiste alors à minimiser la somme des valeurs absolues des résidus sur les débits de la crue $L$ considérée.

L'introduction de contraintes complémentaires :

$P E(i, L)<P B(i, L)$ et/ou $P E(i, L)=$ volume de la crue $L$ est tout à fait possible si les données le permettent.

Jusqu'à présent, la méthode de base et les améliorations proposées avaient surtout été testées sur des données réelles. Les améliorations retenues ne l'étaient donc qu'au vu de résultats de calibrations, s'ils devenaient plus satisfaisants. Mais les échantillons de validation étaient rarement suffisants pour prouver la supériorité définitive d'un nouvel algorithme.

D'autre part, la mise en œuvre de la méthode ne se limite pas à un simple choix d'algorithmes mais comporte un processus décisionnel assez complexe :

- choix des épisodes et du nombre d'épisodes;

- définition du début et de la fin des épisodes;

- choix de la mémoire, ou du nombre d'éléments de la F.T. à identifier;

- choix des algorithmes et des contraintes à appliquer;

- choix de la convergence, etc.

Il était donc souhaitable de la tester sur un ou des jeux de données plausibles mais contrôlées, c'est-à-dire dont on connaisse exactement le modèle sous-jacent, et où l'on ait la possibilité de varier le nombre d'épisodes, leur nature éventuelle (plus ou moins brusque, ou supérieure à des seuils,...) etc. C'est ce qui nous a conduit à des essais en simulation.

\section{Essais en simulation}

La méthode DPFT, comme beaucoup d'autres d'ailleurs, n'a jamais fait l'objet d'une analyse théorique et algorithmique approfondie démontrant sa convergence dans un certain nombre de conditions. La seule tentative, décrite par B. VersianI (1983) sur une suggestion de G. BASTIN, consiste à la rattacher aux méthodes de gradient, dont on connaît les propriétés de convergence. C'est déjà un premier pas réconfortant mais ces méthodes ne prennent pas en compte les contraintes d'inégalité, dont certaines peuvent avoir un rôle déterminant dans la DPFT.

D'autre part, l'analyse mathématique ne permet pas d'aborder les problèmes plus pratiques liés à l'échantillonnage, quand on travaille sur des données en nombre réduit, et les performances de différentes variantes algorithmiques dans un tel contexte de données.

De plus, les données réelles sont en général entâchées d'incertitudes (comme la pluie moyenne sur un bassin) voire d'erreurs (de mesure, de dépouillement, d'archivage, etc.), qui peuvent agir plus ou moins sur les résultats.

Enfin le modèle sous-jacent aux données réelles utilisées peut être plus ou moins éloigné du modèle pour lequel la méthode a été conçue. Il n'est alors pas correct évaluer la méthode sur des données qui ne ressortent manifestement pas du modèle auquel elle se rattache. D'où une réflexion préalable sur les sources d'erreurs, pour décider des essais à réaliser.

\subsection{Mise en œuvre des essais de simulation}

\subsubsection{Sources d'erreur en modélisation}

On peut distinguer un certain nombre de sources d'erreur :

1) Erreur d'adéquation du modèle: On plaque sur la réalité du système physique un modèle dont la structure ne correspond pas à cette réalité. Dans notre cas, hydrologique, il n'est pas toujours sûr que le système naturel ait une "fonction de production" et une "fonction de transfert", ni que cette dernière soit linéaire, comme le modèle le sous-entend.

2) Erreur d'identification: Même si la structure du modèle convient parfaitement, il se peut que l'on n'arrive pas à en identifier l'ordre (i.e. le nombre de paramètres à considérer). Dans notre cas, par exemple, cela concerne la mémoire du système, ou le nombre de pluies efficaces non nulles à considérer.

3) Erreur d'estimation: Ayant déterminé le nombre de paramètres, il faut être capable de retrouver leurs valeurs exactes. Une partie des erreurs d'estimation est due à l'échantillonnage (Période d'ajustement limitée).

4) Erreurs dues aux bruits de mesures: En fait, les valeurs d'entrée et de sortie sont souvent des mesures, plus ou moins bruitées par rapport à la valeur vraie. Idéalement, la méthode d'estimation devra être la moins sensible possible à ces bruits de mesures. 
(1) Gembration

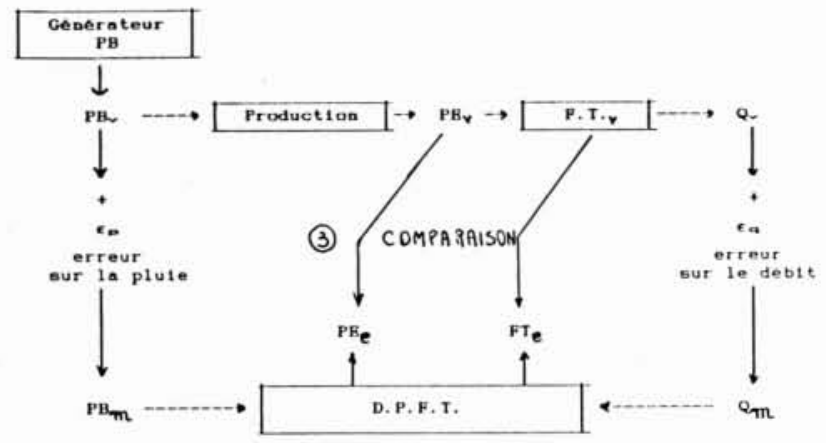

4.

(2) IDEMTIFICATION

Or, si l'on en revient à notre objectif, on remarque qu'il est impossible, sur un système naturel réel, d'assurer que le modèle proposé a bien la même structure que celle, inconnue, du système réel. Dans le cas où cette structure est différente, on ne peut plus parler d'identifier l'ordre "vrai " du système, mais de trouver l'ordre " optimal " du modèle qui permet de minimiser l'erreur de reconstitution.

C'est pourquoi, en vue de tester seulement la méthode d'identification et d'estimation, nous allons choisir, ou plutôt imposer, un système "réel " qui ait la même structure que celle supposée par le "modèle" sousjacent à la méthode.

\subsubsection{Principes des essais en simulation}

Ce système " réel " sera en fait un bassin fictif sur lequel nous allons générer un ensemble de pluies brutes $P_{B}$, reproduisant en gros les caractéristiques des épisodes pluvieux. On postulera ensuite un modèle, non linéaire, de transformation des $P B$ en pluies efficaces $P E$, fonction d'un état initial aléatoire, réinitialisé au début de chaque épisosde. En pratique un modèle assez simple (figure 4) a été utilisé. On fait alors passer à travers ce modèle les $P B_{v}$ " vraies ", et on en tire une série de $P E$ que l'on appellera $P E_{v}$ " vraies ", et qui seront soigneusement mémorisées. On postulera ensuite une fonction de transfert $F T_{v}$ " vraie ", aussi complexe que souhaitée, mais d'intégrale égale à 1 , et elle aussi mémorisée. A l'aide de celle-ci, on fabrique, en partant des $P E_{v}$ "vraies" une série de débits ruisselés $Q_{v}$ "vrais". Eventuellement, on génère, pour chaque épisode, une chronique de débits de base que l'on additionne aux précédents, d'où les débits "vrais ".

Et on génère, comme cela, un nombre assez grand d'épisodes, de longueur et de caractéristiques variées.

La fonction de transfert "vraie" $F T_{v}$ et les pluies efficaces "vraies" $P E_{v}$ serviront ensuite de référence pour les valeurs estimées qu'identifiera la DPFT.

On pourra ainsi évaluer les capacités d'identification de la méthode, d'abord appliquées aux données " vraies " ci-dessus. Puis on ajoutera des erreurs à ces données " vraies", de manière à se rapprocher de données " observées ", incluant les erreurs courantes en hydrologie.

\subsection{Essais sur données exactes}

On dispose donc d'épisodes générés selon le modèle auquel correspond strictement la méthode. Pour tester cette méthode, on ne lui fournira plus que les pluies brutes entrantes et les débits sortants, et on cherchera donc à retrouver la fonction de transfert imposée et les pluies efficaces intermédiaires. Pour cela, on peut faire varier différents paramètres ou certains choix algorithmiques internes à la méthode (cf. § I), les données étant fixées.

\subsubsection{Choix sur les paramètres et les algorithmes}

On a d'abord testé la version de base, la plus proche de celle proposée orginalement, sur un échantillon de 20 épisodes les plus courts (en terme de durée de la pluie).

Dans ce contexte, on a fait varier le choix de la mémoire $K$, utilisée dans l'identification terme à terme de la F.T. (fig. 5, page suivante), avec les conclusions suivantes :

- pour une fonction de transfert donnée, il est souhaitable de choisir un pas de temps tel que la montée de l'hydrogramme représente au moins 3 et plus volontiers 5 pas de temps environ;

- l'identification terme à terme doit porter au moins sur 2,5 fois le temps de montée, mais l'optimum semble être autour de 3 fois ce temps. Au-delà, le lissage de la queue de l'hydrogramme et le fait de renormer l'ensemble de la fonction rendent inutile une identification plus étendue.

On a ensuite testé la vitesse de convergence de la méthode qui s'est révélée rapide au début, plus lente par la suite (au-delà de la $5^{\mathrm{e}}$ itération). On ne poussera donc pas les itérations au-delà de 4 ou 5 .

En fait, c'est plutôt l'application de contraintes qui va confiner la solution, sur la F.T. et surtout sur les PE, à un domaine où les résultats, sans être plus optimaux au sens du critère numérique sur les débits, seront plus réalistes sur les pluies efficaces. Par exemple, l'application de la contrainte :

$$
P E(i, L)<0
$$

est cruciale. Elle intervient sur un pourcentage aussi élevé que $20 \%$ des $P_{E}$ estimées à la première itération.

La contrainte $P E<P B$ a un rôle positif, mais la contrainte de norme sur la F.T. atténue en partie son intérêt.

Or, il apparaît souhaitable de normer la F.T. et de déconvoluer directement au moins à la $1^{\text {re }}$ itération, et éventuellement à toutes, plutôt que de procéder par corrections comme dans la méthode initiale.

Dans le cas de données exactes, les options algorithmiques comme la Ridge Regression et la programmation linéaire se sont révélées un peu inférieures à la version de base. La modélisation ARMAX a donné ici des résultats légèrement supérieures à la méthode de base, et évite aussi le problème du prolongement par lissage - (mais pas celui de la norme) - , au moins quand $m$ et $n$ étaient petits.

\subsubsection{Echantillonnage}

Un autre ensemble d'essais a plutôt porté sur l'échantillonnage. On a d'abord regardé l'effet du nombre 

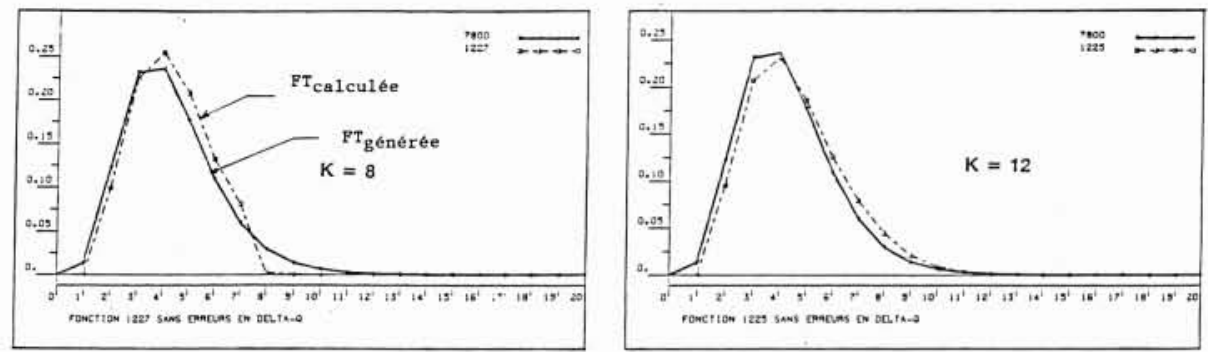

5. Effet de la mémoire $k(k=8,12)$
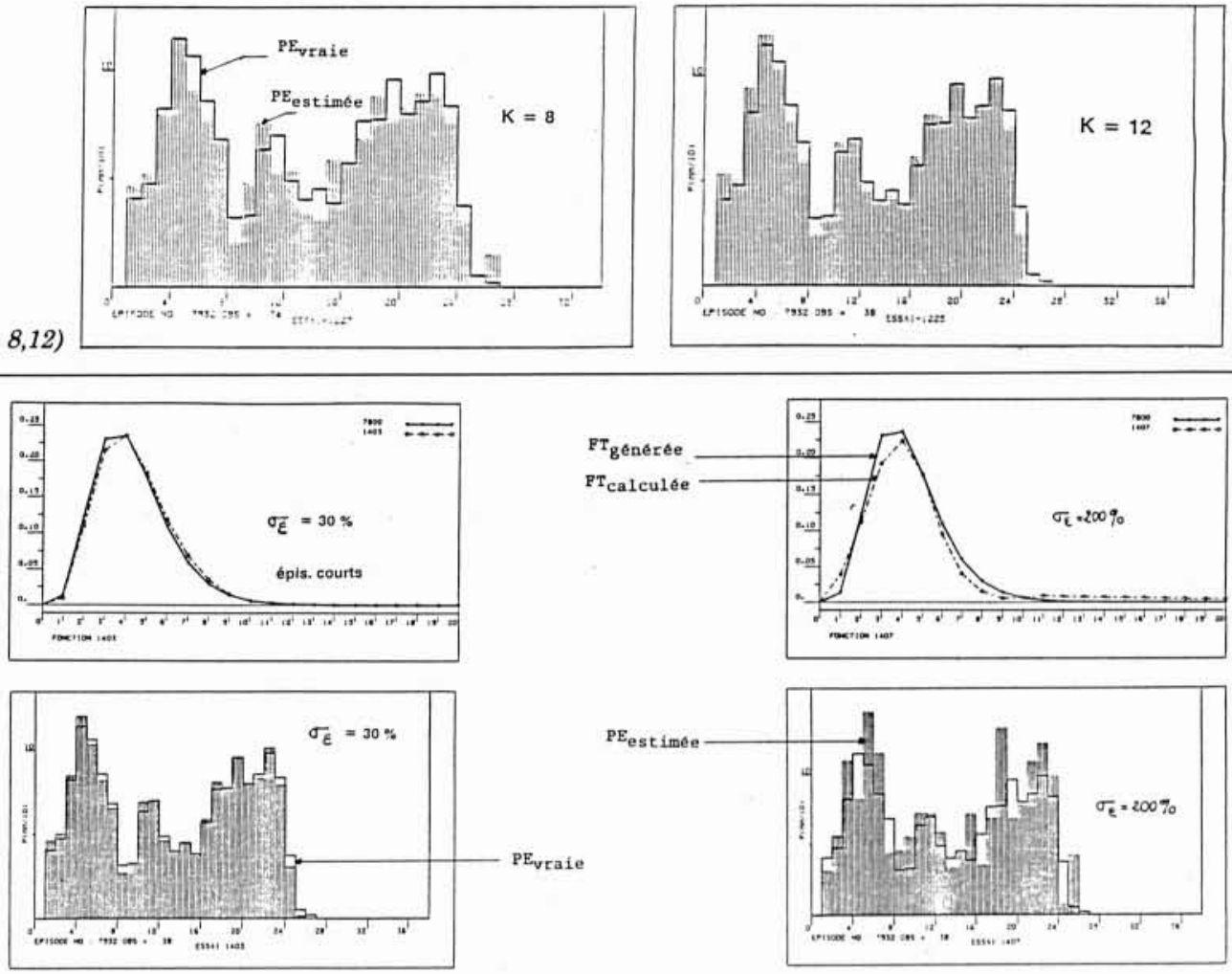

6. Bruit sur la pluie brute
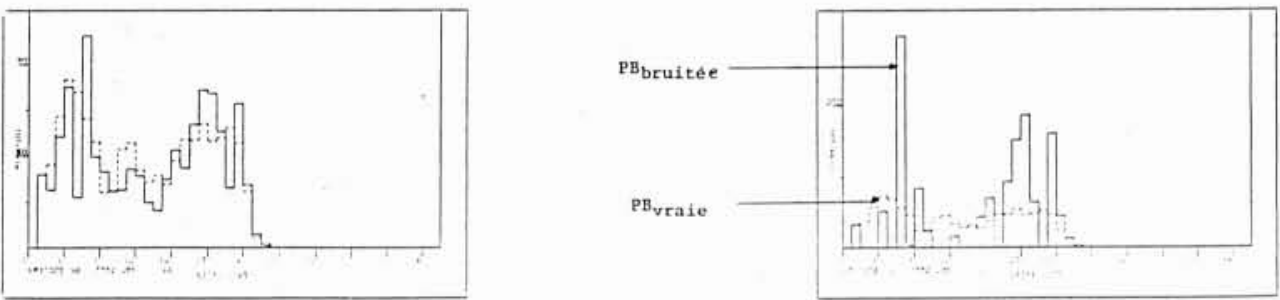

$\sigma_{\varepsilon}=20 \%, 200 \%$
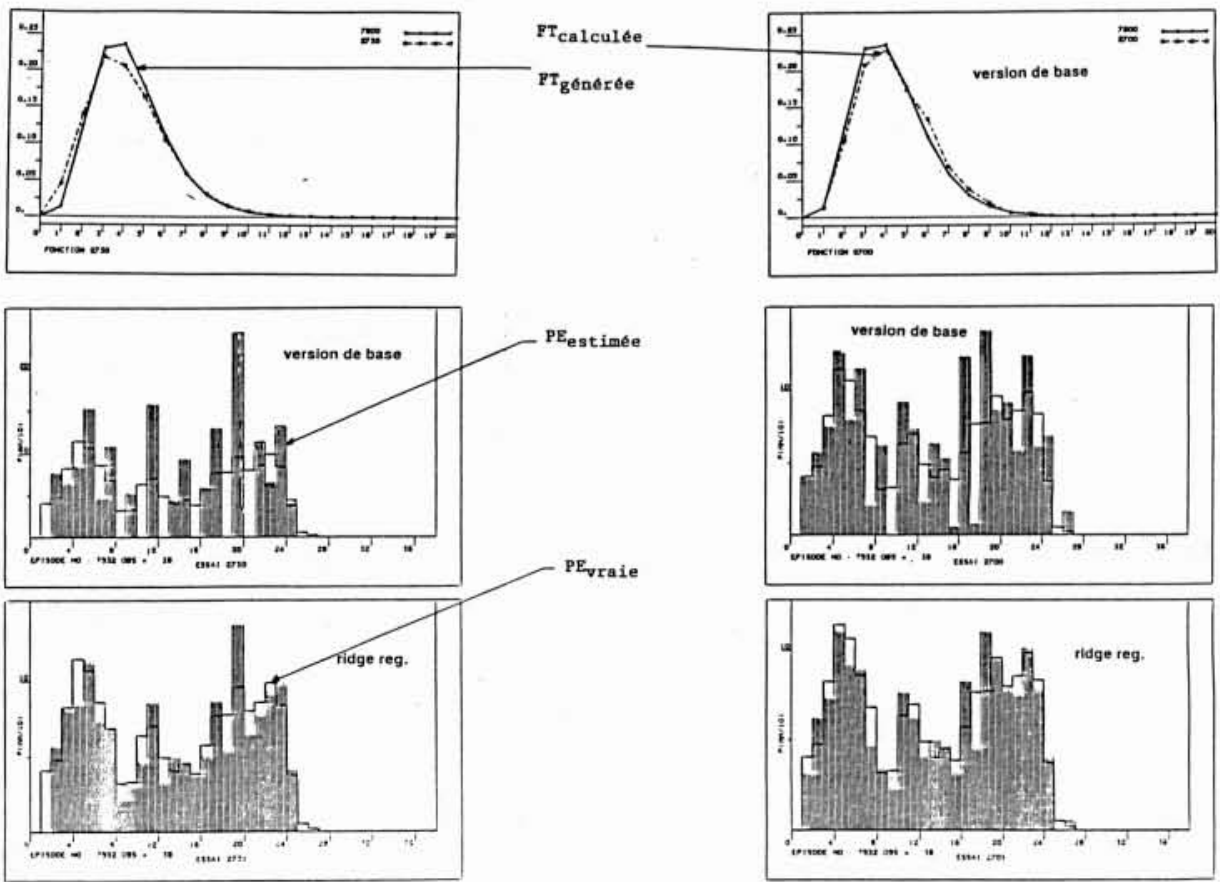
d'épisodes: $(N=5,10,20,30,40)$. On constate que la fonction de transfert identifiée est pratiquement stable pour $N=20$, et que pour $N=10$, voire 5 , on peut l'approcher de façon satisfaisante bien que certains échantillons conduisent encore à de grands écarts.

D'autre part, on note un rôle important de la nonlinéarité dans la relation $P B / P E$. Plus les épisodes sont dans la zone de non-linéarité, plus les résultats s'amenuisent. Or, il faut noter que ce sont sur les épisodes où il y a une grande variation des pluies efficaces, donc de forts débits, que cette non-linéarité se manifeste et qui déterminent donc le comportement de la méthode.

\subsection{Essai avec données de pluies brutes erro- nées}

On a surajouté aux pluies 2 types d'incertitudes : - une erreur d'amplitude, qui représente les bruits de mesure, mais surtout les incertitudes sur la moyenne spatiale dues à l'échantillonnage sur un réseau (cf. LEBEL, 1984, OBLED, 1986, etc.);

- une erreur de "localisation temporelle " de l'épisode, représentant l'incertitude due au réseau sur les heures de début et de fin de l'épisode de pluie brute, mais qui se répercute aussi sur les pluies efficaces.

\subsubsection{Erreur d'amplitude}

On les a représentées par un bruit blanc multiplicatif. $P B_{V}(t)$ vraie est ainsi remplacée par:

$$
P B_{m}(t) \text { mesurée }=P B_{V}(t)(1+\varepsilon(t))
$$

avec $\sigma_{\varepsilon}=.1, .3, .5,1.0,2.0$

Jusqu'à une erreur de $30 \%$, et pour le même jeu d'échantillons $(N=10,20,40)$, la F.T. reste bien identifiée et les $P E$ aussi. Au-delà, des erreurs d'estimation apparaissent sur la $F T$, mais surtout sur les pluies efficaces (fig. 6).

On a aussi testé l'hypothèse selon laquelle la méthode se ramène à une méthode de gradient, pouvant démarrer de n'importe quelle estimation initiale. Pour cela, on a mis comme pluie brute mesurée:

- un bruit blanc pur;

- tel que le volume sur l'épisode soit en espérance identique au volume "vrai ":

$$
P B_{m}(t)=\left(P B_{V} \text { moyen sur l'épisode }\right) x(1+\varepsilon(t))
$$

Mais dans ce cas, la méthode converge vers des valeurs très dégradées de la F.T. et des pluies efficaces, montrant que l'on se démarque, à cause des contraintes intermédiaires, d'une méthode de gradient pur.

\subsubsection{Erreur de chronologie}

On a systématiquement considéré que les épisodes étaient plus longs de 5 ou 10 pas de temps au début et/ou à la fin par rapport à l'épisode « vrai ". Il suffit pour cela d'introduire une pluie brute fictive très petite, ce qui autorise l'algorithme, sur ces pas de temps là, à calculer une pluie efficace.

En fait, les débits étant exacts, on s'aperçoit que, même avec de faibles échantillons, l'algorithme ne dégrade pas la F.T., et ne profite pas de ces degrés de liberté supplémentaires pour y calculer des pluies efficaces significatives.

En conclusion, la méthode est relativement tolérante vis à vis de la pluie brute proposée, c'est-à-dire que si les débits sont corrects, elle reconstitue bien Pluies Efficaces et F.T.

Mais attention, cela ne signifie pas que l'on puisse négliger la mesure convenable des pluies brutes, car la modélisation ultérieure de la fonction de producteur $P B / P E$, non évoquée ici, s'en ressentirait fortement.

\subsection{Erreur sur les débits}

En gardant cette fois les pluies " vraies ", on a introduit sur les débits 2 types d'erreur, qui suivent des modèles multiplicatifs :

$$
\begin{array}{ll} 
& \varepsilon(t)=a_{1} \varepsilon(t-1)+v(t) \\
\text { ou } \quad & \varepsilon(t)=a_{1} \varepsilon(t-1)+a_{2} \varepsilon(t-1)+v(t) \\
\text { avec } & Q_{m}(t)=Q_{V}(t)(1+\varepsilon(t))
\end{array}
$$

Le schéma de Markov d'ordre 1 est plus bruité, tandis que le second est très lisse. Les écarts-types de $\varepsilon$ ont été pris égaux à 3,5 et $10 \%$ et représentent des détarages progressifs de la station de jaugeage en cours de crue, et d'une crue à l'autre (par tirage de $\varepsilon(0)$ ). On n'a pas encore pu tester des bruits plus réalistes (détarages brutaux, débordement en zone inondable, etc.).

On note dans ces essais une grande sensibilité de la F.T. à ce type d'erreur, dès qu'il atteint ou dépasse $5 \%$. Les pluies efficaces estimées s'en dégradent d'autant plus que $\sigma_{\varepsilon}$ augmente.

On retrouve en particulier les fortes oscillations observées dans les essais sur données réelles, où les $P E$ estimées présentent des valeurs, d'un pas de temps sur l'autre, successivement fortes puis faibles ou nulles, tout à fait irréalistes.

Cette instabilité peut alors être corrigée de manière très significative par un algorithme du type Régression en crête, en augmentant la diagonale du système de $10 \%$ environ. Cela introduit certes un biais sur les $P E$ estimées mais les débits eux-mêmes étant faux, le système n'est de toute façon plus tout à fait conservatif.

\subsection{Erreurs combinées sur l'amplitude et la chronologie des pluies et sur les débits}

C'est le cas le plus réaliste. On constate alors que pour retrouver avec une précision raisonnable la $F T$ vraie et surtout les $P E$ vraies, en vue de caler utlérieurement le modèle de production, il faut :

- ne pas dépasser $30 \%$ d'erreur en amplitude sur les pluies. (On notera que ce n'est pas encore à la portée de tous les réseaux et encore moins des radars actuels); - et surtout disposer d'une bonne série de débits connus à moins de $10 \%$ près si possible.

Dans ce cas, proche des conditions réelles, on constate aussi la nécessité d'utiliser la régression en crête pour stabiliser les $P E$ estimées (fig. 7). Il semblerait même que la perturbation à introduire soit proportionnelle à l'incertitude des données.

L'incertitude sur la chronologie de l'épisode (début et fin) n'est pas trop sensible et ne doit pas poser problème, 
même si on calcule de faibles pluies efficaces là où il n'y en a pas.

Par contre, le nombre d'épisodes souhaitables pour la calibration augmente assez sensiblement. Il devient incertain de travailler sur 10 épisodes, et 20 à 40 sont plutôt nécessaires. Accessoirement, il pourrait être intéressant de sélectionner les épisodes. Comme on le constate généralement en traitement de signal, les épisodes pluvieux très variables dans le temps sont préférables (ils excitent mieux la F.T.), mais il s'agit en ce cas des épisodes de pluies efficaces (en effet, un signal de pluie brut même très variable peut être lissé par la fonction de production). Or, il est difficile, en situation réelle, de présumer de la forme du hyétogramme efficace...

\section{Conclusions}

A travers ce travail assez exhaustif mais fastidieux, nous espérons avoir contribué à crédibiliser une méthode dont l'intérêt ne faisait pas encore l'unanimité. Si de nombreuses applications sur données réelles ont donné des résultats intéressants, mais encore insuffisants, quelques applications défaillantes entretenaient le doute.

Nous pensons avoir, sinon démontré, du moins vérifié, par simulation, la pertinence de la méthode proposée par M. DUBAND, et l'intérêt de certaines améliorations proposées par l'IMG, mais sans pouvoir se rattacher complètement à une méthode classique comme celle du gradient. Dans son cadre d'hypothèses, la méthode converge, et vers la vraie solution, en particulier grâce aux contraintes que l'on impose.

La déconvolution directe des pluies efficaces est préférable aux corrections successives, et si les moindres carrés classiques restent supérieurs à la programmation linéaire, la régression en crête, sur données bruitées ou réelles, est à préconiser.

Parallèlement, nous avons pu proposer un certain nombre de règles empiriques de mise en œuvre (mémoire à proposer, choix éventuels des épisodes, etc.) qui devraient guider l'utilisateur occasionnel.

Néanmoins, cette étude, et la méthode elle-même, ne couvrent que la moitié du chemin nécessaire. En effet, on a vu que, même avec des pluies brutes $P B$ assez incertaines, de bonnes mesures de débit permettent une bonne estimation de la $F T$, et surtout, de la série correspondante des pluies efficaces $P E$.

Il reste pourtant à modéliser la relation de production $P B_{\text {mesurée }} / P E_{\text {estimée. }}$ Or, si le $1^{\text {er }}$ membre est fortement entâché d'incertitude, ce modèle fournira des pluies efficaces "modélisées", $P E_{m}$, de mauvaise qualité. Et celles-ci, même transformées par une bonne $F T$, fourniront probablement de mauvaises prévisions de débits... Le schéma de prévision sera alors mauvais, la méthode DPFT n'en restera pas moins pertinente pour autant.

Les efforts à venir devraient donc s'orienter vers cette modélisation de la fonction de production, non plus seulement à partir de considérations physico-théoriques, ce qui a été tenté depuis des décennies, mais à partir de bonnes séries de pluie efficaces estimées dans des bassins convenablement instrumentés.
Les premiers pas de cette démarche sont présentés dans un autre article de cette revue (OBLED et RODRIGUEZ 1987).

Remerciements : Cette étude a été menée grâce au financement du ministère de l'Environnement (contrat SRETIE/ MERE/6003) et de la DATAR Rhône Alpes (Risques Naturels), en collaboration étroite avec la Division technique générale d'EDF.

Mr I. Nalbantis a bénéficié d'une bourse de la Fondation nationale de l'Etat Grec à Athènes.

\section{Références}

BASTIN G., 1984. - Identification de la relation pluie-débit, prévision des débits en temps réel dans Crues et précipitations intenses, I.N.P.G., Fascicule III bis, pp. 1-30.

Delleur J.W. et Ch. OBLed, 1985. - Flash flood forecasting in the Cevennes Region in France: A case study. Proceedings of the Vth World Congress on Water Resources, Bruxelles, Belgique, Pub. IWRA, pp. 871-881.

DUBAND D., 1978. - Fonctions de transfert pluie-débit sur des bassins versants de l'ordre de $1000 \mathrm{~km}^{2}$. Note interne, EDF-DTG, Grenoble.

Dooge J.C.I., 1973. - The linear theory of hydrologic systems, U.S. Dept. of Agriculture Tech. Bulletin, $N^{\circ} 1468$.

Guillot P. et Duband D., 1980. - Fonctions de transfert pluie-débit sur des bassins versants de l'ordre de $1000 \mathrm{~km}^{2}$. La Houille Blanche $\mathrm{N}^{\circ} 4 / 5$.

GuIllor P. et DuBAND D., 1980. - Une méthode de transfert pluie-débit par régression multiple, in Prévisions hydrologiques, Actes du Colloque d'Oxford, avril 1980, Publ. AISH N ${ }^{\circ}$ 129 , pp. 177-185.

HoERl A.E. ET KenNard R.W., 1970. - Ridge Regression: Biased Estimation for Non-Orthogonal Problems Technometrics 12 (1) pp. 55-68.

LEBEL Th., 1984. - Moyenne spatiale de la pluie sur un bassin versant: estimation optimale, génération stochastique, et gradex des valeurs extrêmes. Thèse de Docteur Ingénieur, Grenoble USMG-INPG, 347 p.

NALBANTIS I., 1987. - Identification de modèles pluie-débit du type hydrogramme unitaire: Développements de la méthode DPFT et validation sur données simulées avec et sans erreur. Thèse de Doctorat INPG Grenoble, 493 p.

OBLED Ch., 1986. - Introduction au krigeage pour les hydrologues. $2^{\complement}$ Journées Hydrologiques de l'ORSTOM - Montpellier Septembre 1986, publié dans la collection Colloques et Séminaires, Orstom Ed. 1987, pp. 174-222.

Roche P.A., 1987. - Ouvrage collectif Guide de prévision des crues, Edité conjointement par le Ministère de l'Environnement et la Société Hydrotechnique de France, 324 p.

Salas J.D., Delleur J.W., Yevjevich V. et Lane W.L., 1980. Applied Modelling of Hydrologic Time Series, Water Resources Publications, USA.

Versiani B., 1983. - Modélisation de la relation pluie-débit pour la prévision des crues. Thèse de Docteur-Ingénieur, Grenoble USMG-INPG, 241 p. 
M. SOTTY : Dans la mesure où la pluie brute et la pluie efficace sont indépendantes, la parfaite adéquation de la pluie efficace vraie et de la pluie efficace calculée ne cache-t-elle pas un vice au niveau de la fonction de production?

M. OBLED: Nous avons fait de nombreux tests pour étudier l'influence de la pluie brute, notamment en respectant seulement le volume, et non la chronologie. La méthode fonctionne alors très mal, et il est clair que la pluie efficace varie en fonction de la pluie brute initiale. Schématiquement, l'information chronologique est indispensable et les quantités de pluie doivent être connues avec une précision de 30 à $50 \%$.

M. THIRRIOT: Première question: l'intolérance de la méthode aux bruits sur les débits est-elle à rapprocher des instabilités toujours rencontrées dans la résolution numérique des équations intégrales, et en particulier dans les problèmes de déconvolution? Seconde question : en fonction de votre expérience, que peut-on dire des effets de la non linéarité de la fonction de production? Troisième question : comment peut-on caractériser la sensibilité de la méthode, notamment au niveau de l'estimation de la fonction de transfert?
M. OBLED: Il faut souligner que les critères de calage ne portent ni sur la pluie brute, ni sur la pluie efficace, mais seulement sur les débits: même s'ils sont faux, l'algorithme cherchera à les reconstituer. Les instabilités sont inhérentes à la méthode, à cause notamment des variables explicatives très corrélées, et du fait que l'on travaille sur des produits de grandeurs à estimer. Les contraintes imposées visent justement à limiter ces instabilités. Le problème du calage de la fonction de production n'a pas encore été évoqué. Ce calage est très délicat : les structures du modèle sont mal connues, et les entrées comme les sorties sont affectées d'erreurs de l'ordre de $50 \%$. De ce fait, le modèle de production peut être médiocre, et la méthode donnera alors des résultats médiocres en prévision. Ceci ne remet cependant pas en cause la méthode d'identification. Globalement l'estimation de la fonction de transfert supporte bien l'introduction de données bruitées : l'essentiel des erreurs se reporte sur les pluies efficaces.

M. DUBAND : En complément à la réponse de $\mathrm{M}$. OBLED à $\mathrm{M}$. SoTTY, des études effectuées à la DTG ont montré qu'il fallait un coefficient de corrélation pluie brute/débit d'au minimum 0.6 pour pouvoir initialiser la méthode.

\section{DPFT et innovation : Box, Guillot, Jenkins et les autres}

par C. Thirriot

Depuis longtemps, je flairais que la méthode proposée par Messieurs Duband et Guillot, de la fonction de transfert aux différences premières pour la relation pluie nette-débit était en somme un procédé ARMA tel que proposé par Box et JENKINS. Après la session de la S.H.F. de novembre 1987 et les exposés de MM. Duband et OBLed sur la prévision des crues, j'en suis persuadé.

Dans ce procédé concret, c'est la pluie nette qui joue le rôle de l'innovation. (L'innovation est dans le procédé ARMA le terme d'erreur corrélée $\varepsilon_{t}$ )

Etablissons cela rapidement en l'élargissant à un cas plus général que celui d'une stricte relation pluie-débit et qui reprend notre gestion simpliste de l'information hydrologique pour faire la prévision sur l'Aveyron à la station de Villefranche.

Dans notre exposé sur la comparaison critique des méthodes de prévision des crues, nous avions utilisé une régression multiple pour faire la prévision de l'accroissement de niveau (ou de débit) à la station de Villefranche ce que nous pouvons écrire :

$$
\Delta y_{t}=\sum_{i=1}^{q} \mathrm{a}_{i} \Delta y_{t-i}+\varepsilon_{t}^{\prime}
$$

En fait l'erreur $\varepsilon^{\prime}$, est liée comme $\Delta y_{t-k}$ à l'histoire des pluies à l'amont.

Pour simplifier d'abord, radicalisons la présentation en effacant les termes $\Delta y_{t-i}$ de la pondération. Alors :

$$
\Delta y_{t}=\varepsilon_{t}^{\prime \prime}
$$

C'est alors sous une forme provocante la relation pluie-débit. (Les pluies efficaces sont cachées sous $\varepsilon_{t}^{\prime \prime}$ ).

Evidemment, les $\varepsilon_{t}^{\prime \prime}$ successifs sont liés. Et l'intérêt de la méthode DPFT proposée par MM. DUBAND et Guillot est de présenter très concrètement et de manière physique la corrélation avec l'entrée en scène de la fonction de transfert pluie-débit supposée pérenne du bassin amont. Mais avant de revenir à l'expression formelle des auteurs grenoblois, finissons-en avec la présentation suivant BoX et JENKINS.
Puisque les $\varepsilon_{t}^{\prime \prime}$ successifs sont liés, on va pouvoir écrire qu'ils dépendent de façon linéaire des valeurs successives d'une variable aléatoire qu'on ne connait pas (nous, nous savons bien que c'est la pluie) et qu'on n'a même pas besoin d'identifier physiquement.

$$
\varepsilon^{\prime \prime}{ }_{i}=\sum_{i=0}^{m} b_{i} \eta_{t-i}+\varepsilon_{t}
$$

Nous rajoutons $\varepsilon_{\text {, }}$ pour bien distinguer le caractère physique de la variable aléatoire $\eta_{t-i}, \varepsilon_{t}$ englobant les erreurs de mesure et forcément aussi de modèle.

C'est évidemment alors une naïveté d'écrire à partir de ce qui a été précédemment posé :

$$
\Delta y_{t}=\sum_{i=0}^{m} b_{i} \eta_{t-i}+\varepsilon_{t}
$$

Ceci est donc issu du point de vue désormais classique de Box et JENKINS à peine altéré par l'addition de $\varepsilon_{t}$ erreur non corrélée aux autres erreurs $\eta_{t-1}$.

Revenons maintenant au point de vue DPFT de MM. DUBAND et GuILLot.

Soit $x$ la pluie efficace. Elle est évidemment discrétisée sous forme intégrale au pas d'observation $\Delta t$.

Soit donc $x_{t}$, la pluie sur le pas $\left(t^{\prime}-\Delta t, t^{\prime}\right)$.

Admettons l'hypothèse de l'hydrogramme unitaire représenté par la fonction $h(t)$.

Alors en optique purement déterministe :

$$
\Delta y_{t}=\int_{0}^{t} x(t-) h() d
$$

Pour donner de manière simple une réalité vivante à cette relation, on va discrétiser. Alors :

$$
\Delta y_{t}=\sum_{k=0}^{1} h_{k} x_{t-k} \Delta t+\varepsilon_{t}
$$

$\varepsilon_{t}$ englobe les erreurs de troncature dues à la discrétisation et aussi les erreurs de mesure sur la pluie efficace (on ferme temporairement les yeux sur le fait que ces erreurs de saisie vont introduire une corrélation sur les $\varepsilon_{t} \cdot$ successifs en arguant que les valeurs $x_{t}$, deviennent des variables aléatoires). 
l est la longueur de la fonction de transfert, liée à la mémoire du phénomène physique. Le Professeur OBLED, dans son exposé, a bien insisté sur l'importance des conséquences du choix de ce paramètre du modèle. Les schémas joints donnent une illustration simpliste de quelques cas de discrétisation conservant bien sûr, aire et barycentre.

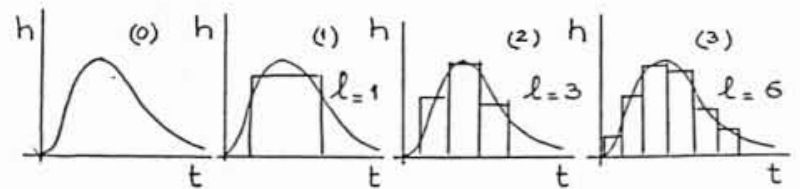

En posant $h_{k} \Delta t=b_{k}$ et $x_{t}=\eta_{t}$, on obtient formellement la même expression à l'issu du cheminement DPFT ou du processus de Box et JeNKINS. La pluie efficace inconnue devient donc le terme d'innovation dans le processus ARMA, innovation parce qu'apportant du neuf dans la considération des erreurs.

Mais n'en restons pas là. Nous voudrions dire que la méthode de MM. DubAND et Guillor a été pour nous l'illustration qui nous a permis de comprendre comment concrètement on gérait le processus de Box et JENKINS. Bien sûr quand les choses sont bien assimilées, tout paraît clair pour ne pas dire évident. Mais avouons bien franchement que ce n'est pas à la première lecture des pages 370 à 420 du célèbre ouvrage "Times Series Analysis * que nous avons parfaitement assimilé la méthode itérative proposée par Box et JENKINS pour résoudre le problème de la détermination des coefficients $b_{i}$ des $t^{\prime}$. Tout s'est élcairé quand nous avons rapproché l'algorithme général de Box et JENKINS de l'algorithme DPFT.

Sur l'exemple concret de la pluie efficace nous avons bien compris qu'il s'agissait alors de la résolution forcément itérative d'un problème non linéaire puisqu'intervenaient les produits $b$ $\eta_{t-i}$ (avec différentes valeurs de $t$ évidemment).

Le fait qu'il s'agisse alors de la résolution d'un système non linéaire permet aussi de bien comprendre l'importance de la quantité d'information.

Il faut encore revenir à l'explication claire et très pédagogique du Professeur OBLed.

Dans le fond, dans l'examen du problème météo hydrologique, on peut parler de pluie efficace sans se soucier de la pluie brute (dans le processus de Box et JENKINS, cette variable aléatoire " amont " n'intervient pas) mais l'observation de la précipitation donne une information qualitative décisive: la longueur du hyétogramme, c'est à dire pour chaque événement pluvieux le nombre des $x_{t}$. inconnus à prendre en considération. Soit $p_{j}$ cette longueur discrète; $j$ est le repère de l'événement pluvieux qui provoque la crue $j$.

Supposont que le fichier de crues dont on dispose comporte $r$ événements $(j \in(1, r))$.

Recensons les inconnues : tisé.

- d'abord les $l$ valeurs $h_{k}$ de l'hydrogramme unitaire discré-

- ensuite pour chaque événement les $p_{j}$ valeurs de pluie efficace soit au total :

$$
\sum_{j=1}^{r} p_{j}=\Pi
$$

Côté observation, on dispose des $\Delta y_{t}$

On peut se poser bien sûr la question de la cohérence des modèles et de l'information observée. C'est un peu ce qu'a fait le Professeur OBLED dans son exposé. Nous ne reviendrons pas là-dessus pour ne pas alourdir cette note qui voudrait être brève. Soit $u_{j}$ le nombre d'observations $\Delta y_{t}$ faites pour l'événement $j$.
En principe si l'hydrogramme unitaire est limité à $\ell$, passé le délai $\ell$ après la fin de la pluie, on ne devrait plus rien observer. Evidemment en fait il $\mathrm{y}$ aura des petits résidus, (peut être pourra-t-on ranger cela dans l'effet du mythique débit de base).

Donc, comme l'indique C. OBLED, pour un jeu rendu cohérent d'observations pour chaque crue, on dispose de $u_{j}$ équations telles que :

$$
u_{j}=p_{j}+\ell
$$

Pour l'ensemble des $r$ événements, on dispose donc d'équation au nombre de :

$$
\Sigma u_{j}=\Sigma p_{j}+r l=\Pi+r l
$$

Le nombre d'inconnues étant $\Pi+l$.

Le système est donc heureusement surdéterminé. S'il y a eu des défaillances dans l'observation, ceci n'est pas très grave tant que leur nombre $d$ reste petit par rapport à $(r-1) /$ ou tout au moins inférieur.

Si on avait $d=(r-1) l$, le système serait isostatique, juste déterminé

Mais évidemment, il vaut mieux qu'il soit surdéterminé (hyperstatique) pour éviter un effet déplorable d'instabilité de la solution.

Ayant le système des $n$ équations individuelles $(\Pi+\ell<n \leqslant \Pi+r l)$, reste alors encore deux étapes.

- le choix d'un algorithme de concentration des équations,

- le choix d'un algorithme de résolution du système qui, bien entendu, reste non linéaire.

Pour la concentration, on peut choisir entre l'utilisation des coefficients de corrélation (DUBAND et GUILLOT) ou bien la méthode plus expéditive des moindres carrés (OBLED). (A propos de cette dernière, nous suggérons l'insertion de coefficients de pondération suivant la confiance accordée à la qualité des observations).

Pour la résolution du système non linéaire résultant, hormis les cas d'un hydrogramme unitaire réduit à un échelon, il faut bien accepter de passer par une méthode itérative. Et là encore, la DPFT de MM. DuBAND et Guillot éclaire très concrètement les possiblités de calage de modèle, de Box et JENKINS.

MM. Duband et Guillot proposent de résoudre successivement deux systèmes linéaires, une fois un système donnant les inconnues $h_{k}$, les $x_{t}$. étant supposés temporairement connus, et à l'étape suivante, on renverse, on détermine les $x_{t}$, les $h_{k}$ ayant les valeurs approchées précédemment déterminées. Bien entendu, on fait l'acte de foi de la convergence.

A notre avis cela pourrait être classé dans la catégorie des directions alternées généralisées plus connues pour la résolution des problèmes aux dérivées partielles multidimensionnels d'espace.

Reste l'initialisation difficile dans les problème général de Box et JENKINS - mais bien facilité dans le problème hydrologique parce qu'on va pouvoir (comme le proposent DUBAND et GuILLOT) utiliser les pluies brutes comme estimations rugueuses des pluies efficaces, ces pluies brutes qui jusqu'à maintenant ne paraissent servir à rien (ce qui est une impression injuste, car elles ont déjà permis de délimiter la longueur des épisodes pluvieux).

En résumé, par discrétisation des informations météorologiques et hydrologiques, la méthode DPFT de MM. DuBAND et GUILLOT est un magnifique exemple concret de modèle à innovation de Box et JENKINS. Le système non linéaire obtenu par produit scalaire du vecteur fonction mémoire (ou hydrogramme unitaire différentiel) et du vecteur hyétogramme efficace est résolu par une méthode de directions alternées suivant le vecteur $\mathrm{H}$ hydrogramme unitaire puis le vecteur $\mathrm{X}$ hyétogramme, suite à initialisation par le vecteur hyétogramme brut observé. 